\title{
Alfabetização, operatoriedade e nível de maturidade em crianças do ensino fundamental
}

\author{
Claudia Araújo da Cunha ${ }^{1}$ \\ Marcionila Rodrigues da Silva Brito \\ Scheila Maria Ferreira e Silva
}

\begin{abstract}
Resumo
Este trabalho teve como objetivo discutir as possíveis relações estabelecidas entre as dificuldades de aprendizagem na escrita, o nível intelectual e maturacional de crianças de segunda série do ensino fundamental. Para tanto, utilizou-se a Escala de Avaliação na Aprendizagem da Escrita (ADAPE), bem como da prova de conservação de comprimento e do teste CAT-H. Os três testes foram aplicados em 60 crianças, de ambos os sexos, de duas escolas da rede pública de ensino. Os coeficientes de correlação por postos de Spearman sugerem que não houve correlações significantes entre os resultados relativos aos três testes e às idades dos sujeitos. Porém, pôde-se encontrar diferença significativa quando comparados os resultados do ADAPE com relação ao sexo feminino ou masculino, encontrando um melhor desempenho dos sujeitos do sexo feminino.

Palavras-Chave: Dificuldade de aprendizagem; Nível de maturidade; Raciocínio lógico.
\end{abstract}

\section{Literacy, operativeness and level of maturity in primary school children}

\begin{abstract}
This paper aims to discuss the relationships established among the learning difficulties in writing and the intellectual and maturity level of the children attending at the second grade of primary school. The Escala de Avaliação na Aprendizagem da Escrita (ADAPE) as well as the length conservation task and the CAT-H were applied to 60 children, of both sexes, from two public schools. The Spearman's rank correlation coefficients suggest that there were not any significant correlation among the data of the three tests and the subjects' ages. It was possible, however, to find a significant difference when comparing the results of the ADAPE to the male and female sexes, the female subjects showing a better performance.

Keywords: Learning difficulties; Maturity level; Logical reasoning.
\end{abstract}

\section{Introdução}

É notório percebermos os inúmeros questionamentos de educadores quanto à dificuldade de aprendizagem na escrita em crianças da rede oficial de ensino. Essas dificuldades podem ser identificadas de diferentes maneiras, seja em razão de um comportamento inadequado, aprendizagem lenta em relação à média das crianças em um conjunto de tarefas, ou até mesmo crianças atrasadas ou em defasagem em exercícios específicos tais como a leitura e/ou a escrita.

Conversas informais com pais e educadores revelam a preocupação constante com o ritmo de uma criança para aprender determinada tarefa se comparada a outra, além das discrepâncias entre o desempenho acadêmico e a aptidão cognitiva (Sisto e cols., 2002). A pergunta premente, então, seria: "Por que existem crianças com desenvolvimento intelectual normal, mas com dificuldade específica em alguma tarefa concreta, como por exemplo, a escrita?" Essas e outras indagações permanecem, muitas das vezes, a rodear os contextos educacionais e porque não dizer socioculturais dos sujeitos cognoscentes.

Ainda segundo Sisto e cols. (2002), inteligência e êxito na aprendizagem estão, historicamente, fortemente associados e têm marcado as teorias psicológicas e a sociedade. Reforça sua afirmativa, enfatizando que é comum ouvir pais, professores, entre outros, chamarem as crianças de inteligentes, quando aprendem ou tiram boas notas na escola ou, ao contrário, lentas e não muito inteligentes, quando tiram notas baixas. Porém, segundo o autor, as medidas de inteligência não informam o aspecto ou aspectos do sistema cognitivo que estejam provocando problemas. Também um diagnóstico da inteligência pode indicar que uma criança tem problemas, mas não qual.

Piaget (apud Flavell, 1996) destaca que a inteligência não é herdada, mas há um modo de funcionamento que nos dá a capacidade de virmos a ser inteligentes por um processo constante chamado invariante funcional, que torna as características do funcionamento intelectual semelhantes às do

Endereço para correspondência:

${ }^{1}$ E-mail: ccunha@triang.com.br

As autoras agradecem ao PIBIC/CNPq o financiamento de uma bolsa de iniciação científica. 
funcionamento biológico. $\mathrm{O}$ intelecto abrange a organização e a adaptação com suas propriedades funcionais de assimilação e acomodação.

$\mathrm{O}$ processo de assimilação se refere à tentativa do sujeito de resolver um problema utilizando elementos já conhecidos por ele. Refere-se àquilo que o sujeito já sabe fazer. Entretanto, quando o sujeito se encontra diante de uma situação nova, ele tem de inovar para modificar seu comportamento, manifestando, pois, uma atividade acomodatícia que visa a um novo ajustamento.

As interações entre organismo-ambiente permitem, então, o funcionamento de esquemas assimiladores que incorporam as necessidades provenientes do organismo em razão deste estar inserido no meio social. Nem só o meio, como diriam os empiristas, nem só o sujeito pré-formado ante as requisições do meio, como postulam os inatistas, constituem para Piaget (1974) a base da aprendizagem construtivista.

Aprendizagem construtivista para Piaget (1974) não é pura aquisição de conhecimento nem também constructos já formados. É uma aprendizagem construída em interações sucessivas entre o sujeito e o objeto de estudo. Num sentido mais amplo, segundo Piaget, aprendizagem é um processo adaptativo se desenvolvendo no tempo, em virtude das respostas dadas pelo sujeito a um conjunto de estímulos anteriores e atuais.

A aprendizagem construtivista acredita que o sujeito acrescenta interpretações próprias às seqüências exteriores que ele apreende. Ele não se limita a registrar tais seqüências exteriores, mas a reestruturá-las na organização de significações. Quem aprende encontrase envolvido por aspectos orgânicos, intelectuais, sociais e afetivos. Esses aspectos reunidos compõem um quadro que, por vezes, explica casos de crianças com dificuldade de aprendizagem (Sisto, 2000).

\section{Dificuldade de Aprendizagem na Escrita}

O estudo das dificuldades de aprendizagem perpassa momentos históricos importantes. Um dos mais relevantes foi, sem dúvida, a passagem dos modelos médicos ou neurológicos que prevaleciam desde o começo do século XIX em favor dos modelos educativos e instrutivos, uma vez que a existência de uma lesão cerebral mínima como agente causador de alterações do comportamento não justificava a ocorrência de outras manifestações neurológicas, necessariamente. Havia, pois, também um favorecimento da biologização do sujeito já que, caso apresentasse algum sintoma, bastaria tomar um medicamento e todo o problema estaria resolvido. Com o advento dos testes psicológicos, houve uma tentativa de se supervalorizar o uso de tais instrumentos. Nesse sentido, o afetivo seria o responsável por condutas do sujeito, até então, inexplicáveis.

As teorias recentes de aprendizagem têm se preocupado com a interação entre o material a ser aprendido e os processos psicológicos necessários para aprender, enfatizando o estudo sobre o modo pelo qual o aprendiz obtém, seleciona, interpreta e transforma a informação (Boruchovitch, 1999). A aquisição do conhecimento, para os psicólogos cognitivos adeptos da Teoria do Processamento da Informação, implica que os indivíduos sejam capazes de ir além do conhecimento factual, em direção ao desenvolvimento de uma capacidade de pensar sobre os próprios pensamentos, em uma metacognição que envolve o pensar sobre as cognições, sobre o comportamento e sobre o próprio processo de aprendizagem, bem como a auto-regulação da aprendizagem. Tal metacognição refere-se ao conhecimento dos próprios processos de conhecer, num planejamento, predição e monitoramento do próprio processo de aprender. Um aluno com desempenho escolar satisfatório, além de ser mais eficaz no uso e na seleção de estratégias de aprendizagem, é sempre capaz de dizer que não entendeu algo, pois ele está constantemente monitorando a sua compreensão.

A alfabetização é um processo bastante complexo, que engendra operações implícitas no reconhecimento de sons que fazem parte de uma palavra, sua transformação em signos e a impressão manual desses signos. A dificuldade de aprendizagem em escrita está, justamente, relacionada ao escrever as palavras deficientemente. Isso significa que os alunos podem reconhecer uma palavra e repetir seus sons, mas não corresponder os diferentes sons às diferentes grafias ou um mesmo som a uma mesma grafia.

Júlio e Brambilla (1996) realizaram uma pesquisa com o intuito de verificar o que pensa o professor sobre a alfabetização em termos genéricos. Foram utilizadas na pesquisa, professoras da primeira série do ensino fundamental. $\mathrm{O}$ instrumento elaborado para tal investigação consta de um questionário composto por quatro questões abertas sobre a visão do professor sobre alfabetização. Os resultados apresentados mostraram que a alfabetização para o professor é basicamente aprender a ler e escrever e obter conhecimento.

Terra e Lana (1996) realizaram uma sondagem de escrita com base nos critérios de escrita pré-silábica, silábica, silábico-alfabética e alfabética. Os resultados evidenciaram que o fato de a criança ter adquirido ou não a consciência do som não determina diretamente o seu fracasso ou seu sucesso na aquisição da linguagem escrita. Além do fato desta aprendizagem ser multifatorial, algumas crianças também demonstraram uma evolução na escrita sem ter adquirido a consciência 
dos sons. O contrário também foi evidenciado: outras que não evoluíram na escrita e, concomitantemente, adquiriram a consciência dos sons.

Santos e Marturano (1999) atentam para o estudo da dificuldade de aprendizagem, enfocando as questões sociais, emocionais ou de comportamento como possíveis fatores associados ao fracasso escolar. Foram avaliados 41 adolescentes de ambos os sexos, com idade média de 13 anos e nove meses. Formaramse dois grupos, um de nove adolescentes encaminhados a serviços de Saúde Mental por apresentarem atualmente dificuldades severas de ajustamento; e outro com dez adolescentes com dificuldades mínimas de ajustamento. Comparando os grupos, observou-se maior acúmulo de fatores negativos antecedentes, familiares e pessoais, no grupo de adolescentes com pior ajustamento atual.

\section{As Operações Lógicas}

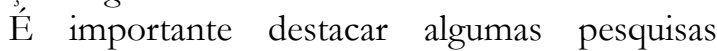
encontradas na literatura sobre a aquisição do raciocínio lógico diante de situação de aprendizagem, visto que a aprendizagem pode ser vista como algo construído ao longo de um processo, definindo categorias de evolução cognitivas, indo de um nível menos evoluído a um mais evoluído (Sisto, 2000). Ortega, Alves e Rossetti (1995) realizaram um trabalho com o intuito de investigar, em contextos construtivistas e não-construtivistas, o raciocínio lógico de crianças por intermédio de um jogo de regras (Jogo da Senha). Participaram como sujeitos 40 crianças que cursavam as primeiras e segundas séries do primeiro grau, 20 de uma escola pública com uma proposta educacional construtivista e 20 de uma escola particular sem a referida proposta, ambas na cidade de Vitória-ES. Os resultados obtidos mostraram que na escola que possui a proposta educacional construtivista, o desempenho cognitivo das crianças foi significativamente superior nas duas situações experimentais.

Strauss e Ilan (1975) pesquisaram a relação entre a tarefa de conservação de comprimento e os conceitos de velocidade. O grupo experimental passou por uma sessão de treinamento. Nela, os sujeitos produziram julgamentos contraditórios, baseados na velocidade relativa de carros em situações hipotéticas. Os resultados indicaram que os sujeitos que apresentaram noção de conservação na prova de comprimento no pré-teste modificaram seus raciocínios intuitivos em conceitos de velocidade no pós-teste.

Pires (1988) desenvolveu um estudo com o propósito de investigar as relações entre o acesso às estruturas operatórias concretas e o desempenho escolar das crianças no que se refere à aprendizagem inicial da leitura e escrita. Para isso, selecionou uma classe com 20 crianças de baixa renda. Foram avaliados quanto ao nível de desenvolvimento cognitivo nas operações de classificação de desempenho de tarefas em leitura/escrita, por meio de duas provas (mudança de critério ou dicotomia e de adequação da inclusão de classes), aplicadas em etapas diferentes. Os resultados demonstraram que os sujeitos que conseguiram superar as formas elementares de classificação passando do nível figural ao não figural ou ao nível das classificações hierárquicas revelaram bons desempenhos em leitura/escrita.

\section{Dificuldade de aprendizagem e fatores cognitivos/emocionais/ afetivos}

Sisto e cols. (2002) afirmam não ser suficiente ter grande inteligência para realizar uma boa aprendizagem, sendo necessário que esta seja acompanhada de uma estrutura de personalidade sadia e emocionalmente madura. Isso significa que o nível de maturação de uma criança para a aprendizagem depende das inter-relações estabelecidas entre os fatores intelectuais, emocionais e biológicos.

Yaegashi (1999) realizou uma pesquisa com 200 crianças com idade entre 6 e 10 anos, alunas de primeira e segunda série de escolas públicas, todas provenientes de famílias de baixo nível socioeconômico. Para a coleta de dados utilizou-se dos seguintes instrumentos: teste de desempenho escolar, prova das matrizes progressivas de Raven, provas operatórias, provas de criatividade, Teste gestáltico visomotor de Bender e Prova de Rorschach. No que diz respeito aos resultados, nas provas operatórias, na prova de criatividade, bem como no teste gestáltico visomotor de Bender, não houve diferenças significativas entre os grupos. Quanto aos indicadores de distúrbios emocionais e de lesão cerebral também não houve diferença significativa, porém foi constatado que, nos grupos da segunda série, os resultados revelaram a falta de estabilidade emocional, ansiedade, timidez e agressividade.

Mais recentemente, Martinelli (2002) afirma que o aspecto afetivo é um importante elemento a se considerar quando se pretende compreender o processo de aprendizagem dos indivíduos. Situações como o abandono, a separação dos pais, a perda de entes queridos, um ambiente desfavorável à manifestação afetiva, a depreciação são variáveis intervenientes no processo de aprendizagem. A autora também aponta que a experiência repetida de insucesso pode levar ao caminho do fracasso escolar e ao abandono do processo de escolarização. Recentemente, na tentativa de compreender mais acerca das dificuldades de aprendizagem, as relações entre o aspecto cognitivo e o afetivo têm sido novamente enfatizadas.

Sisto e cols. (2002) defende que a aprendizagem de leitura e escrita constitui uma tarefa fundamental na educação. Esta tarefa tem sido objeto de análise e 
investigação de muitos estudiosos que tentam explicá-la, como também investigam medidas práticas para solucionar as possíveis dificuldades de alunos e professores. A literatura mostra que a análise das relações entre dificuldade de escrita e leitura e a força da integridade do ego, apesar de sua constatada importância, é escassa. Assim, esse autor realizou um estudo cujo objetivo era avaliar até que ponto diferentes graus de integridade do ego estariam relacionados ao desempenho em provas de ditado, no ensino fundamental.

Com uma amostra de 56 crianças da primeira série do ensino fundamental e de nível socioeconômico baixo, aplicou-se o teste desiderativo para avaliar a força do ego do indivíduo e três tipos de ditado para avaliar os níveis de dificuldade na escrita. Os resultados permitiram constatar que um grande número de sujeitos apresentou fragilidade de ego do tipo moderada ou severa e um número grande de crianças, presença de fortes conflitos emocionais. Além disso, evidenciou-se a enorme dificuldade das crianças no que diz respeito à escrita. Os resultados mostraram que erros no ditado estavam relacionados significativamente com o grau de força de ego. Quanto mais forte era o ego da criança, menor era a média de erros no ditado de palavras e letras. O que reafirma a relação entre o fracasso escolar e fatores emocionais como ansiedade, depressão e outros.

Partindo do pressuposto que a dimensão afetiva permeia todo o processo ensino-aprendizagem, e que é importante o professor estar bem fundamentado e seguro para atuar como mediador do conhecimento, Sadalla \& Silva (1996) realizaram discussões a respeito da prática cotidiana do professor, tais como o desenvolvimento infantil, o fracasso escolar, a postura do professor em sala de aula e a teoria interacionista. Os resultados apontaram que a falta de compreensão por parte do professor, de que a relação afetiva está estreitamente ligada à capacidade de aprendizagem do aluno, é um dos fatores que contribuem para o fracasso escolar.

Com base em tais resultados, uma pergunta permanece: até que ponto um sujeito classificado como não-conservador apresentará dificuldades na escrita, configurando um perfil de dificuldade de aprendizagem leve? E seu nível de maturidade encontrar-se-á regredido para sua idade? Essas questões implicam discussões acerca de como se efetivou a alfabetização de um grupo de crianças de 2a série, a construção do conhecimento das mesmas, assim como o nível de maturidade que oportuniza a execução de tarefas desse porte. Dentro dessa perspectiva, a presente pesquisa teve como objetivo investigar as relações estabelecidas entre a dificuldade de aprendizagem da escrita, o nível cognitivo e o maturacional, em sujeitos entre 8 e 10 anos, diante de três instrumentos, Escala de Avaliação de Dificuldades na Aprendizagem da Escrita (ADAPE), a prova de conservação de comprimento preconizada por Piaget e o teste projetivo CAT-Humano (Bellak \& Bellak, 1965).

\section{Método}

\section{Sujeitos}

Participaram da pesquisa 60 sujeitos, alunos da 2 a série do ensino fundamental, de duas escolas da rede pública de ensino da cidade de Uberlândia-MG, sendo uma escola municipal e outra estadual. Foram aplicados os testes em 29 crianças da escola estadual, 14 do sexo feminino e 15 do sexo masculino. Na escola municipal, aplicaram-se os testes em 13 meninas e 18 meninos, totalizando 31 crianças.

\section{Instrumentos}

O texto da escala de Avaliação de Dificuldades na Aprendizagem da Escrita (ADAPE) foi construído e validado por Sisto e cols. (2002), defendendo alguns critérios como: palavras usuais no cotidiano escolar das crianças; uma mesma palavra poderia conter mais de uma dificuldade; e deveria haver pelo menos um terço de palavras trissílabas e/ou polissílabas. O texto é constituído por 114 palavras, com 60 delas apresentando algum tipo de dificuldade classificada como encontro consonantal, dígrafo, sílaba composta e sílaba complexa, e 54, não. Cada uma das palavras foi considerada um item ou unidade de medida para efeitos de pesquisa.

A prova de conservação de comprimento aqui aplicada foi uma adaptação da prova original a partir da deformação de linhas a comparar (Piaget, 1976). Foram utilizados os seguintes materiais: a) quatro palitos de madeira medindo $7 \mathrm{~cm}$ de comprimento por $0,8 \mathrm{~cm}$ de largura, denominados palitos grandes, e nove palitos pequenos, medindo $4 \mathrm{~cm}$ de comprimento por $0,8 \mathrm{~cm}$ de largura, denominados palitos pequenos, e b) uma folha de registro elaborada para esta prova. Nela foram anotadas as respostas de cada sujeito e o tempo de duração.

O teste projetivo CAT-H (Bellak \& Bellak, 1965) foi realizado mediante a apresentação de dez pranchas com figuras humanas, no qual cada criança deveria elaborar uma breve história com base na cena representada por cada uma das pranchas. Essas histórias deveriam conter os sentimentos e pensamentos dos personagens, o que gostam de fazer e o que estava acontecendo com eles em cada cena apresentada.

\section{Procedimento}

A aplicação dos testes foi feita em duas etapas. Primeiramente, foi aplicado o ditado proposto por Sisto e cols. (2002), realizado na própria sala de aula. Depois, em sala apropriada, foram aplicados a prova de conservação de comprimento e o teste CAT-H, administrados individualmente. 
A aplicação do ditado foi feita pela professora da classe de 2a série, juntamente com um experimentador, depois de instruída para informar aos alunos que eles iriam fazer um ditado, que seria ditada uma palavra de cada vez e nenhuma delas seria repetida e, por isso, precisariam prestar bastante atenção. Para a correção dos ditados, cada palavra foi considerada uma unidade e qualquer erro ortográfico ou ausência de palavra foi considerada erro, assim como acentos e letras maiúsculas e minúsculas indevidas, sendo a soma dos erros a pontuação de cada criança. Quanto à classificação: até 20 erros (sem dificuldade de aprendizagem); entre 21-49 erros (início de indicação de dificuldade de aprendizagem); 50-79 erros (dificuldade de aprendizagem leve) e 80 ou mais erros (dificuldade de aprendizagem média).

A prova de conservação de comprimento foi iniciada colocando-se quatro palitos grandes alinhados em uma reta, construída pelo experimentador. A reta que a criança construísse deveria conter sete palitos pequenos para que ficasse do mesmo comprimento que a reta do experimentador. Em seguida, foram feitas mais quatro transformações, solicitando à criança que dissesse se existe igualdade no comprimento das duas estradas, acrescentado-se o pedido para que a criança justificasse sua resposta. Quanto aos critérios de classificação da prova, três tipos de respostas foram considerados: a) ausência de conservação, quando os sujeitos não afirmaram a igualdade das retas; b) reações intermediárias: quando as crianças oscilavam entre a conservação e a não-conservação e c) resposta de conservação, quando o sujeito coordenou as operações de partição e de colocação ou deslocamento. A aplicação do teste foi de aproximadamente 10 minutos.

As instruções dadas para a aplicação do CATHumano foi a seguinte: "Hoje iremos brincar de contar histórias. Você as contará olhando um desenho e nos dirá o que está acontecendo e o que as pessoas estão fazendo.” A análise interpretativa está dividida em dez variáveis, quais sejam, tema principal; herói; quais figuras são visualizadas e, diante delas, como a criança reage; qual a concepção de mundo; figuras, objetos e circunstâncias externas introduzidas; objetos ou figuras omitidos; natureza das ansiedades; conflitos significativos; severidade do superego; integração do ego (nível de maturidade emocional). Nesta última, mede-se o nível de maturidade da criança, caracterizando-o como aquém, além ou normal, de acordo com a idade da mesma. Este item foi priorizado na análise dos dados da presente pesquisa. O tempo de duração foi de aproximadamente 1 hora para cada criança. Foi anotado o tempo de duração para cada história e o tempo de latência, ou seja, período de reação do sujeito ao observar a prancha, antes da dissertação da sua história.

\section{Resultados}

Os resultados obtidos estão nas Tabelas 1, 2 e 3. Todos esses dados foram computados, utilizando o programa de análise estatística SPSS. Cada resultado de cada teste e todas as variáveis envolvidas (sexo, idade, ordem de aplicação) foram colocados no programa para serem correlacionados.

Tabela 1 - Ditado: instrumento de avaliação de dificuldade de aprendizagem na escrita

\begin{tabular}{lccc}
\hline Número de Erros & $\begin{array}{c}\text { Número de Crianças } \\
\text { Escola Estadual }\end{array}$ & $\begin{array}{c}\text { Número de Crianças } \\
\text { Escola Municipal }\end{array}$ & $\begin{array}{c}\text { Classificação } \\
\text { Categoria }\end{array}$ \\
\hline até 20 erros & 01 & 05 & cat 0 \\
21 a 49 erros & 18 & 19 & cat 1 \\
50 a 79 erros & 10 & 04 & cat 2 \\
80 ou mais & & 03 & cat 3 \\
\hline
\end{tabular}

Tabela 2 - Prova de Conservação de comprimento

\begin{tabular}{lcc}
\hline Classificação & Número de Crianças & Número de Crianças \\
& Escola Estadual & Escola Municipal \\
\hline Não conservadora & 23 & 17 \\
Intermediária & 04 & 13 \\
Conservadora & 02 & 01 \\
\hline
\end{tabular}

Tabela 3 - Teste CAT-H

\begin{tabular}{lcc}
\hline Classificação & Número de Crianças & Número de Crianças \\
& Escola Estadual & Escola Municipal \\
\hline Além do esperado & 01 & 00 \\
Dentro do esperado & 20 & 11 \\
Aquém do esperado & 08 & 20 \\
\hline
\end{tabular}


Com o objetivo de verificar a existência ou não de correlações significantes entre os resultados relativos ao ditado, à prova de conservação de comprimento, à avaliação do teste CAT-H e às idades dos sujeitos, foi calculado o coeficiente de correlação por postos de Spearman (Siegel, 1975) aos valores das variáveis, combinados dois a dois. O nível de significância foi estabelecido em 0,05 , em uma prova bilateral. Os resultados estão na Tabela 4. De acordo com os resultados mostrados na Tabela 4, não foram encontradas correlações significantes entre as variáveis analisadas.

Tabela 4 -Valores de $r_{s}$ do Coeficiente de Correlação por Postos de Spearman

\begin{tabular}{lcc}
\hline Variáveis Analisadas & Valores de $\mathrm{r}_{\mathrm{S}}$ & Probabilidades \\
\hline CAT x ditado & $-0,0024$ & 0,986 \\
CAT x idade & $-0,1168$ & 0,374 \\
CAT x Piaget & $-0,1701$ & 0,194 \\
Ditado x idade & $-0,0139$ & 0,916 \\
Ditado x Piaget & 0,0316 & 0,810 \\
Idade x Piaget & 0,1809 & 0,167 \\
\hline
\end{tabular}

Devido ao fato de 30 sujeitos terem sido submetidos, primeiro à prova de Piaget e, depois, ao teste CAT-H, e a prova de Piaget ter sido aplicada em segundo lugar para os outros 30 sujeitos, foi usado o coeficiente de correlação por postos de Spearman, a esses dois grupos, para a verificação da existência ou não de correlações significantes entre eles. O nível de significância foi estabelecido em 0,05 , em uma prova bilateral. Os resultados estão na Tabela 5. De acordo com esses resultados, não foram encontradas correlações significantes entre as variáveis analisadas.

Tabela 5 - Valores $r_{s}$ do Coeficiente de Correlação por Postos de Spearman

\begin{tabular}{ccccc}
\hline Variáveis & Valores de $\mathrm{r}_{\mathrm{s}}$ & Probabilidades & Valores de $\mathrm{r}_{\mathrm{s}}$ & Probabilidades \\
\cline { 2 - 5 } Analisadas & Piaget/ CAT-H & Piaget/ CAT-H & CAT-H/ Piaget & CAT-H/ Piaget \\
\hline CAT x ditado & 0,0669 & 0,725 & $-0,0729$ & 0,702 \\
CAT x ditado & $-0,0746$ & 0,695 & $-0,1833$ & 0,319 \\
CAT x ditado & $-0,3053$ & 0,101 & $-0,0305$ & 0,873 \\
CAT x ditado & 0,2701 & 0,149 & $-0,1726$ & 0,362 \\
CAT x ditado & 0,2972 & 0,111 & $-0,2098$ & 0,266 \\
CAT x ditado & 0,0829 & 0,663 & 0,3188 & 0,086 \\
\hline
\end{tabular}

Com o intuito de verificar a existência ou não de diferenças significantes entre os resultados obtidos pelos elementos do sexo masculino e do sexo feminino, foi aplicado o teste U de Mann-Whitney (Siegel, 1975), aos resultados obtidos com o ditado, teste CAT-H e prova de Piaget. O nível de significância foi estabelecido em 0,05 , em uma prova bilateral. Os resultados estão demonstrados na Tabela 6. De acordo com esses resultados, foram encontradas diferenças significantes entre os resultados relativos ao ditado, tendo os elementos do sexo feminino obtido valores mais elevados do que os do sexo masculino.

Tabela 6 - Probabilidades obtidas quando da aplicação do teste de Mann-Whitney

\begin{tabular}{lcc}
\hline Variáveis & Probabilidades & Valores de U \\
\hline Ditado & $0,0173^{*}$ & 310 \\
CAT-H & 0,6114 & 421 \\
Piaget & 0,4355 & 406 \\
\hline
\end{tabular}

$\left({ }^{\circ} \mathrm{P}<0,05\right.$

\section{Discussão e Considerações Finais}

Em se tratando de variáveis relativas ao cotidiano escolar, privilegiando-se aspectos que envolvem o cognitivo e o afetivo, faz-se mister tecer algumas considerações. A presente pesquisa procurou explorar o nível de operatoriedade, de maturidade emocional e da dificuldade de aprendizagem na escrita de um grupo de sujeitos da rede oficial de ensino. Tal objetivo configura-se a partir do momento em que somos sabedores da incidência alarmante de crianças que não se alfabetizam nem na primeira nem na segunda série do ensino fundamental. Especificamente, procuraram-se estabelecer possíveis relações entre a 
tríade alfabetização-operatoriedade-maturidade dentro de um contexto que suscita dúvidas, tais como "Por que existem crianças com desenvolvimento intelectual normal mas com dificuldade específica em alguma tarefa concreta, como por exemplo, a escrita?”

As relações estabelecidas não apresentam uma ordem direta de acontecimento. Isso significa que não necessariamente uma criança que não apresente dificuldade de aprendizagem na escrita seja também conservadora no conteúdo conservação de comprimento, bem como se encontre num nível de maturidade pelo menos dentro do esperado para sua faixa etária. $\mathrm{Na}$ realidade, podemos encontrar diferentes tipos de condutas. Sisto e cols. (2002) destaca a presença de fatores não só orgânicos no diagnóstico de problemas de aprendizagem. Mas acrescenta a esse os fatores específicos (transtornos na adequação perceptivomotora), fatores psicogênicos (aspectos afetivos e emocionais) e, por fim, fatores ambientais (possibilidades que o meio fornece). Dentro dessa perspectiva, é importante considerarmos que mesmo provenientes de níveis socioeconômico-culturais empobrecidos, cada qual reage de dada maneira mediante estímulos diversos. Isso porque, não necessariamente, aquele que sofre conflitos emocionais apresenta dificuldade de aprendizagem na escrita ou em qualquer outro conteúdo. Talvez por se tratar de uma dificuldade circunstancial, medida num determinado momento histórico, se diagnosticado mais adiante pode não apresentar a tendência de dificuldade de aprendizagem leve ou mediana.

O mesmo podemos falar quanto a operatoriedade. Esta pode ser ausente, intermediária ou presente em vários conteúdos ministrados. O sujeito faz uma opção de escolha no seu sistema cognitivo, corroborando a premissa de que fatores endógenos e exógenos contribuem para a sua mudança de comportamento, desde que haja motivação e desejo pelo objeto do aprender, significando, pois, que cada experiência é dotada de características próprias, únicas e internas. Em realidade, estamos lidando com alunos que aprendem necessariamente de forma pessoal, intransferível e interna. Nesse sentido, conhecimento, indubitavelmente, é distinto de desempenho, pois é possível termos conhecimento, por exemplo, sobre a língua inglesa, mas isso não garante que tenhamos um bom desempenho quando submetidos à execução de uma canção na referida língua. Não somente nascemos com um saber fazer, como diria Piaget, mas também somos seres que influenciam e são influenciados pelo ambiente e, assim sendo, interagem com o meio de forma dinâmica e construtiva, acrescentando novas idéias, crenças e comportamentos a realidades, muitas das vezes, repetitivas e, por que não dizer, reprodutoras de reflexões já desatualizadas do contexto atual e moderno do qual fazemos parte. Por isso, talvez possamos explicar que dois sujeitos expostos às mesmas condições socioambientais, possam comportar-se de formas tão distintas na família, na escola e em outros contextos sociais.

Novas competências devem ser desenvolvidas junto ao alunado, visando a organização e direção de situações de aprendizagem. Assim, a busca de recursos cognitivos, objetivando atingir uma meta, deve ser o ponto principal para a formação de educadores, cônscios de sua importância no desempenho acadêmico dos seus alunos.

Os resultados da pesquisa evidenciaram que a maioria dos sujeitos pesquisados $(n=40)$ apresentaramse não conservadores no teste de conservação de comprimento, 31 crianças encontram-se dentro do esperado, segundo o nível de maturidade emocional, e 37 crianças apresentam início de indicação de dificuldade de aprendizagem na escrita. Isso corrobora as idéias acima colocadas de que não necessariamente um nível de desenvolvimento cognitivo operatório designa um nível de maturidade emocional dentro do esperado ou além deste, assim como crianças sem indício de dificuldade de aprendizagem na escrita.

Quando procuramos realizar possíveis relações entre as variáveis estudadas (aplicação do ditado, prova de conservação de comprimento e o teste projetivo CAT-H) com a idade dos sujeitos envolvidos, as correlações estatísticas não se mostraram significantes, apontando para o fato de que as idades dos sujeitos variavam somente em poucos meses, não contribuindo com o aparecimento de possíveis relações significativas.

A ordem de aplicação dos testes, por sua vez, também não evidenciou correlações relevantes, talvez pelo fato de que enquanto a aplicação do teste de conservação de comprimento durou aproximadamente 10 minutos, o teste CAT-H demorou mais que o esperado para ser aplicado em sua totalidade. Isso pode ter levantado alguns questionamentos: até que ponto o teste CAT-H cansou algumas crianças que, talvez por isso, não tenham relatado histórias criativas e dinâmicas em algumas das pranchas? Se isso de fato aconteceu, a ordem de aplicação dos testes não teria ficado prejudicada como um todo?

Já com relação à significância demonstrada pelos resultados obtidos pelas meninas na aplicação do instrumento ADAPE, se comparadas aos resultados dos meninos, algumas hipóteses relacionadas a comentários oriundos da comunidade escolar justificariam tal fato devido ao maior capricho e cuidado quando solicitadas a executarem alguma atividade em específico. Pais e demais responsáveis remetem-se com freqüência a questões do tipo: “a letra de menina é mais bonita que 
de menino"; "Meninas são mais cautelosas que meninos", entre outras.

Apesar de sabermos que tais ditados populares não acontecem necessariamente nessa proporção, uma pergunta permanece: "Teriam as meninas maior aptidão para a escrita se comparadas aos meninos?" Essas e outras indagações dão margem a outras pesquisas que devem enfocar tais variáveis.

\section{Referências}

Bellak, L. \& Bellak, S. S. (1965). Teste de apercepção infantil com figuras humanas. São Paulo (SP): Mestre Jou.

Boruchovitch, E. (1999). Estratégias de aprendizagem e desempenho escolar: considerações para a prática educacional. Psicologia: Reflexão e Critica, 12(2), 361-376.

Flavell, J. H. (1996). A psicologia do desenvolvimento de Jean Piaget. 5. ed. São Paulo: Livraria Pioneira.

Júlio, A. A. \& Brambilla, L. H. (1996). A percepção do professor sobre o processo de Alfabetização. Anais do III Congresso Nacional de Psicologia Escolar, Campinas, PUCCAMP.

Martinelli, S. C. (2002). Os aspectos afetivos das dificuldades de aprendizagem. Em F. F. Sisto, E. Boruchovich, L. D. T. Fini, R. P. Brenelli \& S. C. Martinelli (Orgs.). Dificuldade de aprendizagem no contexto psicopedagógico. Petrópolis: Vozes.

Ortega, A. C., Alves, R. M. \& Rossetti, C. B. (1995). Raciocínio lógico e jogo de regras: contextos construtivista e não-construtivistas. Arquivos Brasileiros de Psicologia, 3(47), 105-112, jul./set.

Piaget, J. \& Gréco, P. (1974). Aprendizagem e conhecimento. Rio de Janeiro: Freitas Bastos.

Piaget, J. (1976). A equilibração das estruturas cognitivas. Rio de Janeiro: Zahar.

Pires, Y. M. C. (1988). O desenvolvimento de estruturas operatórias concretas e a aprendizagem inicial da leitura/escrita em crianças de baixa renda. Arquivos Brasileiros de Psicologia, 2, 63-72.
Sadalla, A. M. F. \& Silva, R. C. (1996). A dimensão afetiva da alfabetização: a contribuição do psicólogo escolar ao cotidiano do professor. Anais do III Congresso Nacional de Psicologia Escolar, Campinas, PUCCAMP.

Santos, L. C. \& Marturano, E. M. (1999). Crianças com dificuldade de aprendizagem: um estudo de seguimento. Psicologia, Reflexão e Crítica, 12(2), 377-394.

Siegel, S. (1975). Estatística não-paramétrica, para as ciências do comportamento. São Paulo: McGraw-Hill do Brasil.

Sisto, F. F. (2000). Defasagem de desenvolvimento e transferência de aprendizagem construtivista. Psicologia, Reflexão e Crítica, 13(1), 25-32.

Sisto, F. F., Oliveira, G. C. \& Fini, L. D. T. (2000). Leituras de Psicologia para a formação de professores. Petrópolis: Vozes.

Sisto, F. F., Boruchovich, E., Fini, L. D. T., Brenelli, R. P. \& Martinelli, S. C. (Orgs.). (2002). Dificuldade de aprendizagem no contexto psicopedagógico. 2. ed. Petrópolis: Vozes.

Strauss, S. \& Ilan, J. (1975). Length conservation and speed concepts: Organizational disequilibrium training between concepts. Journal of Educational Psychology, 67(4), 470-77.

Terra, A. P. C. \& Lana, M. (1996). A consciência dos sons da fala exerce influência no processo de aprendizagem da leitura e da escrita? Um estudo exploratório sob o olhar da Psicologia Escolar. Anais do III Congresso Nacional de Psicologia Escolar - Prevenção e Saúde Mental na Educação, Campinas, PUCCAMP.

Yaegashi, S. F. R. (1999). As dificuldades de aprendizagem nas séries iniciais. Em F. F. Sisto (Org.). O cognitivo, o social e o afetivo no cotidiano escolar. (p. 167-195). Campinas: Papirus.

Recebido em agosto de 2003

Reformulado em novembro de 2003 Aprovado em dezembro de 2003

Sobre as autoras:

Claudia Araújo da Cunha é psicóloga, doutora em Psicologia Educacional pela Faculdade de Educação da UNICAMP e professora adjunta da Faculdade de Psicologia da Universidade Federal de Uberlândia.

Marcionila Rodrigues da Silva Brito é psicóloga, mestre em Psicologia Clínica pela PUCCAMP e professora adjunta da Faculdade de Psicologia da Universidade Federal de Uberlândia.

Scheila Maria Ferreira e Silva é graduanda em psicologia, cursando o 8o período na Universidade Federal de Uberlândia e bolsista pelo CNPq por dois anos consecutivos. 